\title{
Geographien zwischen Diskurs und Praxis - Mit Wittgenstein Anknüpfungspunkte von Diskurs- und Praxistheorie denken
}

\author{
C. Baumann, A. Tijé-Dra, and J. Winkler \\ Institut für Geographie, Friedrich-Alexander-Universität Erlangen-Nürnberg, Nuremberg, Germany \\ Correspondence to: C. Baumann (christoph.baumann@fau.de)
}

Received: 4 November 2014 - Revised: 15 May 2015 - Accepted: 7 July 2015 - Published: 4 September 2015

\begin{abstract}
Kurzfassung. Current discussions about a practice turn in human geography raise questions about whether discourse analytical approaches are focusing too strongly on symbolic representations. However, practicetheoretical conceptions privilege the analysis of everyday practices, discussing issues of embodiment and materiality. Following the considerations of Ludwig Wittgenstein, we assume certain connections between these two fields of study and explore the links between discourse and practice theory by employing the Wittgensteinian concepts of language-games and rule-following. Focussing on the discourse theory of Laclau and Mouffe and the practice theory of Schatzki, we show to what extend both theories are informed by Wittgenstein's work and can be seen as different types of language-games analyses. We highlight the similarities and discuss the differences in the basic interests of these theories. Finally, we introduce an integrative "Wittgensteinian approach" that is illustrated by two empirical examples of geographical inquiry - practices of a new rurality and rap-music in stigmatized banlieus.
\end{abstract}

\section{Einleitung}

Diskurs und Praxis gehören zu wichtigen theoretischen und analytischen Bezügen in der Humangeographie. Im Kontext des linguistic/cultural turns (Glasze und Pütz, 2007) sind seit der Jahrtausendwende zahlreiche diskursanalytische Studien in Anschluss an Foucault oder Laclau und Mouffe in der deutschsprachigen Geographie entstanden. Mit dem verstärkten Aufgreifen der praxistheoretischen Überlegungen Bourdieus, des Pragmatismus und aktuell Schatzkis zeigt sich bei geographischen Arbeiten in jüngerer Vergangenheit auch ein practice turn (Everts et al., 2011).

Diskurs- wie praxisorientierte Arbeiten sind jeweils auch als Kritiken an bestimmten konzeptionellen Entwicklungen der Humangeographie zu sehen. Die vor allem im Diskussionszusammenhang der Neuen Kulturgeographie eingenommenen diskursanalytischen Perspektiven teilen die beispielsweise in der handlungsorientierten Sozialgeographie geäußerte Kritik an raumessentialistischen Vorstellungen, fokussieren dabei aber weniger die Rolle einzelner intentional handelnder Akteure. Vielmehr geht es um die überindividuelle
Konstitution und Repräsentation von Räumlichkeit in Form symbolischer Ordnungen, die zumeist text- bzw. zeichenanalytisch herausgearbeitet werden. Praxistheoretische Konzeptionen legen ihren Schwerpunkt weniger auf symbolische Repräsentationen, als auf Praktiken in ihren materiellen und situativen Einbettungen, welche mit ethnographischen Methoden in den Blick genommen werden. Der practice turn steht auch im Zusammenhang mit der aktuell diskutierten more-than-representational geography (Lorimer, 2005), die die einseitige Fokussierung auf Repräsentationen kritisiert und sich, verstanden als eine "geography of what happens“" (Thrift, 2008:2), vorranging für Alltagspraktiken in ihren performativen, körperlichen und affektiven Facetten interessiert.

Wie verhalten sich nun Diskurs(theorie) und Praxis(theorie) zueinander? Steht ein praxisorientierter Blick der Diskursforschung entgegen? Ist das Verhältnis zwischen Diskurs und Praxis gar dichotomisch angelegt, z.B. als sprachlich, zeichenhaft, abstrakt, strukturell, immateriell vs. nicht-sprachlich, körperlich, konkret, situativ, materiell? Nein. Beide Theoriefamilien sind keine verriegelten, sondern 
offene Denkgebäude, die zwar jeweils Schwerpunkte setzen, aber Anschlüsse bieten, das vermeintlich ,Andere“ mitzudenken. Das zeigt sich an einer Reihe von Arbeiten, die an den Schnittstellen diskurs- und praxistheoretischer Annahmen agieren - sowohl in allgemeinen sozialwissenschaftlichen wie auch in geographischen Debatten. Solchen Arbeiten geht es darum, die praktische Dimension und Materialität des Diskursiven deutlicher zu machen und diskursanalytische Verfahren stärker ethnographisch auszurichten (Kap. 2).

In diesem Schnittstellenbereich von Geographien zwischen Diskurs und Praxis verorten wir unseren Aufsatz, in dem wir Anknüpfungspunkte bestimmter diskurs- und praxistheoretischer Überlegungen erkunden. Dabei konzentrieren wir uns auf zwei Theorieangebote, die in aktuellen humangeographischen Arbeiten eine wichtige Rolle spielen, aber bislang weitgehend unabhängig voneinander diskutiert werden: die Diskurstheorie Laclaus und Mouffes sowie die Praxistheorie Schatzkis. Beide setzen zwar unterschiedliche Schwerpunkte und nutzen dabei ein spezifisches Vokabular, teilen aber einen ähnlichen Blick auf die soziale Welt. Dies, so argumentieren wir, lässt sich gerade auch darauf zurückführen, dass beide Ansätze jeweils stark von Ludwig Wittgensteins Spätphilosophie zum Sprachspiel geprägt sind. Indem wir beide Entwürfe als Spielarten einer „Sprachspieltheorie“ deuten, arbeiten wir deren Verhältnis systematisch heraus und zielen darauf ab, konzeptionelle Anschlussstellen wie auch unterschiedliche Erkenntnisinteressen herauszustellen. Darauf aufbauend schlagen wir eine integrative Perspektive vor, bei der die Fragestellung ,How do empty signifiers matter?“ den Fluchtpunkt empirischer Studien ,zwischen Diskurs und Praxis“ darstellt (Kap. 3). Inwiefern eine solche Perspektive für humangeographische Analysen nutzbar gemacht werden kann, skizzieren wir anschließend anhand zweier kurzer, erkundender Beispiele (Kap. 4).

\section{2 Überblick: Diskursforschung und ihr Praxisverständnis}

Die Diskursforschung (nach Foucault) ist kein homogenes Feld (Keller, 2011a). Auch wenn die verschiedenen Ansätze eine sozialkonstruktivistische Grundperspektive teilen, situationsübergreifende Muster sozialer Bedeutungsproduktion untersuchen und die Zentralität von Sprache betonen, sind sie von unterschiedlichen Annahmen geprägt. Dies betrifft etwa Fragen nach dem Status des Subjekts, den Grenzen des Diskurses oder dem Verhältnis von symbolischer Ordnung und Materialität (z.B. Van Dyk et al., 2014). Ein wichtiger Kreuzungspunkt dieser Debatten ist das Verständnis von Praxis. In einer vielzitierten Stelle der „Archäologie des Wissens“" schreibt Foucault (1973:74), die Diskursanalyse sei

eine Aufgabe, die darin besteht, nicht - nicht mehr - die Diskurse als Gesamtheiten von Zeichen [...], sondern als Praktiken zu behandeln, die systematisch die Gegenstände bilden, von denen sie spre- chen. Zwar bestehen Diskurse aus Zeichen; aber sie benutzen diese Zeichen für mehr als nur zur Bezeichnung der Sachen. Dieses mehr macht sie irreduzibel auf das Sprechen [parole] und die Sprache [langue]. Dieses mehr muss man ans Licht bringen.

Foucault, der sich vor eindeutigen Positionierungen sperrte und seine Diskursbegriffe häufig modifizierte, entwickelte keine systematische „Praxistheorie des Diskurses“, aber eröffnete einen Auslegungsspielraum für diskursorientierte Bestimmungen von Praxis.

Dabei dominierten zunächst sehr sprachorientierte Herangehensweisen. Ein Grund dafür ist der Rezeptionsweg der Foucault'schen Ideen. Im deutschsprachigen Kontext waren es mit Jürgen Link (1982) und Siegfried Jäger (1989) zunächst Germanisten, die entsprechende Überlegungen aufnahmen. Da z.B. Jäger diskursive Praxen als „Sprechen und Denken auf der Grundlage von Wissen“ (2011:82) begreift, konzipiert er seine Kritische Diskursanalyse auch explizit textanalytisch.

Ergänzt werden die linguistischen Rezeptionen seit den 1990er Jahren von stärker sozialwissenschaftlichen Lesarten, die sich um ein erweitertes Diskursverständnis bemühen ${ }^{1}$ : etwa durch die Bezugnahme auf weitere Konzepte Foucaults wie „Dispositiv“ (z.B. Bührmann und Schneider, 2008) und „Gouvernementalität“ (z.B. Bröckling et al., 2000) oder auch durch die Kombination der Überlegungen Foucaults mit anderen Theorieangeboten wie beispielsweise der Distinktionstheorie Bourdieus (Diaz-Bone, 2010).

Diese unterschiedlichen Wege gehen einher mit einem unterschiedlichen Verständnis von (diskursiver) Praxis. Während z.B. wissenssoziologische Ausrichtungen von diskursiven Praktiken (,typisch realisierte Kommunikationsmuster [...] in einem Diskurszusammenhang“), diskursgenerierenden Praktiken (,Muster für Handlungen, die in Diskursen artikuliert werden) und diskursexternen Praktiken (,von Diskursen unabhängige Handlungsvollzüge“; Keller, 2011b:228, 257) sprechen, begreifen stärker poststrukturalistisch geprägte Ansätze das Diskursive als praxisinhärent und heben die Trennung von Diskurs und Praxis auf (z.B. Wrana und Langer, 2007; Wrana und Ott, 2010; Wrana, 2012). ${ }^{2}$

\footnotetext{
${ }^{1}$ Ein sehr umfang- und facettenreiches Bild der aktuellen Diskursforschung liefern Angermüller et al. (2014).

${ }^{2}$ Die AutorInnen kritisieren eine kategoriale Unterscheidung von diskursiven/nicht-diskursiven Praktiken durch die Diskussion verschiedener Referenzen (z.B. Wrana und Ott, 2010: Foucault, Althusser, Butler, Laclau und Mouffe; Wrana, 2012: Foucault, Butler, Derrida, Bourdieu). Dabei zeigen sie Facetten einer ethnographisch informierten, poststrukturalistischen Diskursanalyse auf und liefern sehr interessante Fallstudien aus den Erziehungswissenschaften. Wir teilen deren poststrukturalistischen, situierteren Zugang zur Diskursanalyse ,[j]enseits der Unterscheidung diskursiver und nicht-diskursiver Praktiken“ (Wrana und Ott, 2010) und erachten dabei gerade das praxistheoretische Vokabular Wittgensteins und Schatzkis als sehr aufschlussreich und gewinnbringend.
} 
Im Laufe der vergangenen Dekade entstanden auch in der deutschsprachigen Humangeographie unterschiedliche Spielarten diskursanalytischer Studien (Glasze und Mattissek, 2014). Im Kontext einer Neuakzentuierung der Politischen Geographie als eine Kritische Geopolitik fanden diskursanalytische Ansätze eine breitere Rezeption (z.B. Wolkersdorfer, 2001). Zentral hierbei ist die Analyse von raumbezogenen Repräsentationen (geopolitische Leitbilder), deren Produktion häufig in einer akteurszentrierten Perspektive Politikern, Medien oder Wissenschaftlern zugesprochen wird. Die Diskursanalyse fungiert z.T. als Ergänzung einer handlungsorientierten Sozialgeographie. Ein Diskurs ist demnach eine praxislegitimierende symbolische Ordnung, die primär durch sprachliche Handlungen vollzogen wird und strategisch eingesetzt werden kann.

Poststrukturalistische Ansätze haben ein anderes akzentuiertes Diskursverständnis. Das betrifft zum einen die Zurückweisung der Analysekategorie eines intentional handelnden Subjekts, zum anderen eine Erweiterung des Diskurs- und Praxisbegriffs. In Anschluss an Laclau und Mouffe konzipieren Glasze und Mattissek (2009a:11f.) Diskurse als ,überindividuelle Muster des Denkens, Sprechens, Sich-selbstBegreifens und Handelns sowie der Prozesse, in denen bestimmte Vorstellungen und Handlungslogiken hergestellt und immer wieder verändert werden“. Diskurse beschränken sich so weder auf ausschließlich sprachliche Ebenen, noch sind sie extern der - sondern als - Praxis zu denken. Methodisch überwiegen allerdings textorientierte Zugänge, mit denen hegemoniale Ordnungen herausgearbeitet werden (Kap. 3.2). Daneben sind Entwürfe entstanden, welche sprachfokussierte Studien um eine diskursorientierte Analyse von Bildern oder Karten ergänzen (z.B. Miggelbrink und Schlottmann, 2009).

Im Kontext der practice/performative/material turns kommen Vorschläge für eine deutlichere Materialisierung und praktische Situierung diskurstheoretisch informierter Konzepte auf. Zwei Beispiele: Wucherpfennig und Strüver (2014; vgl. Strüver und Wucherpfennig, 2009) interpretieren die Arbeiten Foucaults performativitätstheoretisch, um in Anschluss an Butler auch nichtsprachliche Akte der Signifikation sowie deren Räumlichkeit stärker zu berücksichtigen. Aus feministischer Perspektive betonen sie die KoKonstitution von Identität, Raum und Gesellschaft. Diese vollzieht sich über die Materialisierung symbolischer Ordnungen, die vergeschlechtlichte Körper sozial positionieren und oft nur bei Irritationen sichtbar und anfechtbar werden (z.B. wenn Frauen ,unweiblichen“ sportlichen Praktiken an „männlich“ kodierten Orten nachgehen). In Auseinandersetzung mit den critical geopolitics mahnt Müller (2008, 2009) an, dass sich diese zu sehr auf die Dekonstruktion großer Zusammenhänge konzentrieren und das ,Kleine“ auBer Acht lassen (2008:324). Diskurstheoretische Überlegungen mit Bourdieus bildungssoziologischen Konzepten kombinierend, legt er den Fokus darauf, wie ,great power iden- tities“ hergestellt und von Studierenden einer russischen Diplomatenhochschule inkorporiert werden.

Arbeiten wie diese zeugen von aktuellen Bemühungen einer stärkeren „Praxeologisierung“ diskursorientierter Konzeptionen in der Humangeographie. Diese greifen wir auf, indem wir im Folgenden explizit die konzeptionellen Anschlussstellen zwischen der Diskurstheorie Laclaus und Mouffes und der Praxistheorie Schatzkis, mit Wittgenstein, diskutieren.

\section{Mit Wittgenstein Anknüpfungspunkte der Diskurstheorie Laclaus und Mouffes und der Praxistheorie Schatzkis denken}

Beide hier verhandelten Theorieangebote sind wichtige Referenzpunkte aktueller Arbeiten der Kultur- und Sozialgeographie. Der poststrukturalistische Blick Laclaus und Mouffes auf überindividuelle Muster der Bedeutungskonstitution hat bereits zahlreiche humangeographische Studien angeleitet. Ebenso werden in jüngerer Vergangenheit verstärkt Überlegungen des Sozialphilosophen und Geographen Schatzki genutzt, um eine praxistheoretische Perspektive auf das Geographie-Machen anzulegen (siehe die Theme Issue innerhalb der vorliegende Artikel publiziert ist). Wir schlagen eine Brücke zwischen den beiden Konzeptionen, in dem wir im Folgenden ein „Netz von Ähnlichkeiten, die einander übergreifen und kreuzen“ (Wittgenstein, 2008:\$66) ${ }^{3}$ herausarbeiten. Rahmen und Motiv dieser Auseinandersetzung liefert uns die Spätphilosophie Ludwig Wittgensteins, die für beide Theorieangebote einen wichtigen Bezug darstellt. ${ }^{4}$

\subsection{Wittgensteins Sprachspiel-Konzept}

Ludwig Wittgenstein beschäftigt sich in seinen Philosophischen Untersuchungen ausgiebig mit (Sprach-)Praxis. Im Zentrum dieser Auseinandersetzung steht der Begriff Sprachspiel, den er folgendermaßen illustriert:

A führt einen Bau auf aus Bausteinen; es sind Würfel, Säulen, Platten und Balken vorhanden. B hat ihm die Bausteine zuzureichen, und zwar nach der Reihe, wie A sie braucht. Zu dem Zweck bedienen sie sich einer Sprache, bestehend aus den Wörtern: „Würfel“, „Säule“, „Platte“, „Balken“. A ruft sie aus; B bringt den Stein, den er gelernt hat, auf diesen Ruf zu bringen. (ebd.:§2)

Ich werde auch das Ganze: der Sprache und der Tätigkeiten, mit denen sie verwoben ist, das ,Sprachspiel“" nennen. (ebd.:§7)

\footnotetext{
${ }^{3}$ Der Konvention folgend zitieren wir Wittgenstein in Paragraphenform.

${ }^{4}$ Diese Wittgenstein-Lektüre hat zur Konsequenz, dass wir andere konzeptionelle Bezüge der Theoretiker eher ausklammern. Etwa Derrida und Lacan (Laclau und Mouffe) oder Dewy und Heidegger (Schatzki).
} 
Wir wollen das Sprachspielkonzept nun in vier wesentlichen Dimensionen erörtern.

\subsubsection{Gebrauchstheorie der Bedeutung}

Wittgenstein betrachtet Sprache nicht als ein abstraktes, durch eindeutige Regeln fixiertes System, sondern als eine Praxis. „Die Bedeutung eines Wortes ist sein Gebrauch in der Sprache“ (Wittgenstein, 2008:§47). Das Wort „Balken“ hat keine Bedeutung an sich, sondern nur in praktischen $\mathrm{Zu}$ sammenhängen wie beispielsweise dem Bau eines Hauses oder einer Lehrveranstaltung, in der Diagrammtypen erklärt werden. Wittgensteins Überlegungen sind als eine radikale Kritik an der zeitgenössischen Philosophie zu verstehen, die für ihn zu sehr in der Sphäre der Metaphysik, also jenseits der Alltagspraxis, denkt (ebd.:§116). Er vollzieht eine alltagspraktische Wende und konzentriert sich mit zahlreichen Beispielen - das Bauarbeiterbeispiel ist eines der bekannteren - auf die praktischen Kontexte von Sprache. Aber mehr noch:

\subsubsection{Sprachliche und nicht-sprachliche Tätigkeiten}

Die Forderung „Sieh den Satz als Instrument an, und seinen Sinn als seine Verwendung!“ (ebd.:§421) wurde gerade in der Sprachpragmatik ausbuchstabiert. Wittgensteins Überlegungen sind aber mehr als sprachphilosophisch. Sie gehen „weit darüber hinaus, indem sie Nichtsprachliches zur notwendigen Bedingung für das Verständnis des Sprachlichen erklär[en]" (Schulte, 2001:146). Sprachspiele sind eben nicht - wie der Begriff nahelegt - Spiele, die ,nur“ aus Sprache bestehen, sondern sie sind „das Ganze“. Das oben zitierte Bauarbeiterbeispiel bezieht sich auf Begriffe, aber auch auf Tätigkeiten wie das Rufen und das Steinereichen. Das Sprachspiel ist also ein ,Zusammenhang zwischen der Verwendung sprachlicher Ausdrücke und eingefleischten Handlungsweisen“ (ebd.).

\subsubsection{Abrichtung, Regelfolgen und das Primat der Praxis}

Zentral für Wittgensteins Ansatz ist das praktische Erlernen. Diesen Sozialisationsprozess bezeichnet er als eine Abrichtung (z.B. Wittgenstein, 2008:§6). Individuen werden abgerichtet, in bestimmten Situationen in einer bestimmten Weise „Tätigkeiten zu verrichten“, „Wörter zu gebrauchen“ sowie auf eine bestimmte Weise, auf die Worte des anderen zu reagieren“ (ebd.). Das Erlernen der Bedeutung eines Wortes oder einer nicht-sprachlichen Tätigkeit ergibt sich vor allem aus deren Anwendung im Kontext einer Lebensform (ebd.:§241). Man wird abgerichtet, an Sprachspielen teilnehmen zu können, indem man die Fähigkeit erwirbt, in ähnlichen Situationen (d.h. in Situationen, die als ähnlich gedeutet werden) verstehbar bzw. anschlussfähig zu handeln. Die Abrichtung spiegelt sich in einem „Weiterwissen“ (u.a. ebd.:\$154), einem Wissen, das in bestimmten Situationen wahrscheinlich macht, dass sozial gehandelt und in diesem Sinne Regeln gefolgt wird. ${ }^{5}$ Genau mit diesem „Regelfolgen" setzt sich Wittgenstein ausgiebig auseinander. Er fragt: „Was nenne ich ,die Regel, nach der er vorgehe“?” (ebd.:\$82). Wittgenstein wendet sich gegen ein Verständnis, welches Regeln fasst als ,abstrakte oder mentale Entitäten, die unabhängig [...] bestimmten, was als korrekte und inkorrekte Befolgung gilt“" (Puhl, 2011:92). Regeln sind für ihn keine autonomen, extern die Praxis determinierenden Repräsentationen, sondern konstitutiver Teil der Praxis (ebd.; Bloor, 2001). Ob eine Regel „richtig“" angewendet wird, lässt sich nur im praktischen Regelfolgen erkennen und ist je nach Situation immer wieder eine Frage der Deutung dessen, was als Regelfolgen oder -bruch gilt. Wittgensteins zentrale Kritik am autonomen Regelkonzept ist das sogenannte Regelregressargument (vgl. Römpp, 2010:100ff.), das er folgendermaßen illustriert:

Nimm an, ich erkläre: „Unter ,Moses ‘ verstehe ich den Mann, wenn es einen solchen gegeben hat, der die Israeliten aus Ägypten geführt hat, wie immer er damals geheißen hat und was immer er sonst getan, oder nicht getan haben mag“. - Aber über die Wörter dieser Erklärung sind ähnliche Zweifel möglich wie die über den Namen „Moses“ (was nennst du „Ägypten“, wen „die Israeliten“, etc.?). Ja, diese Fragen kommen auch nicht zu einem Ende, wenn wir bei Wörtern wie „rot“, „dunkel“, „süß“, angelangt wären. (Wittgenstein, 2008:§87)

Da jede Regel weitere (Meta-)Regeln benötigt (in dem Beispiel die Verwendungsregeln von Begriffen), kann sie Tätigkeiten, seien sie sprachlich oder nicht-sprachlich, nie letztbegründen. Wittgenstein führt aus: „Jede Deutung hängt, mitsamt dem Gedeuteten, in der Luft" (ebd.:§198). So ist es nicht eine autonome Regel, die Bedeutungen begründet, sondern das praktische Tun, ,welche[s] am Grunde des Sprachspiels liegt“ (Wittgenstein, 1970:§204). Und genau ,[d]arum ist ,der Regel folgen“ eine Praxis“(Wittgenstein, 2008:\$202).

\subsubsection{Offenheit und Geschlossenheit des Sozialen}

Wittgenstein geht von einer prinzipiellen Offenheit des Sozialen aus. Da es keine Regeln geben kann, die in jedem Falle Tätigkeiten genau bestimmen, ist die Praxis offen und bietet Möglichkeiten der Variation. Wenngleich Sprachspiele für ihn „nichts Festes, ein für allemal Gegebenes“ (ebd.:§23), sind, so denkt er sie doch partiell verfestigt. Zwar kann jede

\footnotetext{
${ }^{5}$ Wittgenstein deutet hier auch an, dass durch Prozesse der Abrichtung zu einem bestimmten Praktizieren hin andere PraktikenAlternativen verdrängt werden. Dies verweist auch auf eine Machtdimension des Sprachspiel-Konzepts. Macht im Sprachspiel hat keinen festen Ort und ist nicht die Ressource eines Individuums, sondern eher in einem „Ensemble von Handlungen, die sich gegenseitig hervorrufen und beantworten“ (Foucault, 1987:251) zu verorten.
} 
Tätigkeit mit jeder denkbaren Regel über Deutung in Einklang gebracht werden, manche Deutungen dürften in der Praxis jedoch schwerer legitimierbar sein als andere, da sich Routinen des (Regel-)Gebrauchs verfestigen. Jemand richtet sich nach „Wegweisern“, weil es einen „ständigen Gebrauch, eine Gepflogenheit, gibt“ (Wittgenstein, 2008:§198), sich nach diesen zu richten. Ein Sprachspiel kann daher gedacht werden als ein Muster, welches sich variierend wiederholt. Wittgenstein fasst das Verhältnis zwischen der Offenheit und Geschlossenheit in seiner Auseinandersetzung mit dem Begriff „Weltbild“ folgendermaßen:

Die Sätze, die dies Weltbild beschreiben, könnten zu einer Art Mythologie gehören. Und ihre Rolle ist ähnlich der von Spielregeln, und das Spiel kann man auch rein praktisch, ohne ausgesprochene Regeln, lernen. [...] Man könnte sich vorstellen, daß gewisse Sätze in Form der Erfahrungssätze erstarrt wären und als Leitung für die nicht erstarrten, flüssigen Erfahrungssätze funktionierten; und daß sich dies Verhältnis mit der Zeit änderte, indem flüssige Sätze erstarrten und feste flüssig würden. [...] Die Mythologie kann wieder in Fluß geraten, das Flußbett der Gedanken sich verschieben. (Wittgenstein, 1970:\$95-97)

Diese „Sedimentierungsüberlegungen“ buchstabiert er nicht weiter aus. Sie liefern aber, um im Bilde zu bleiben, nicht zuletzt ein Flussbett für poststrukturalistische Diskurstheorien.

\subsection{Die Diskurstheorie Laclaus und Mouffes}

Die PolitikwissenschaftlerInnen Laclau und Mouffe entwickeln in einer kritischen Auseinandersetzung mit dem Marxismus eine Diskurstheorie (z.B.: 2006, 127-188, vgl. dazu auch Glasze und Mattissek, 2009b), die gerade auch von Wittgenstein beeinflusst ist. Wittgensteins ,neue Art der Theoretisierung“, so Mouffe (2008:69), sei von nicht zu unterschätzender Bedeutung.

\subsubsection{Diskurs als Sprachspiel}

Diese wichtige Rolle wird bei der Einführung ihres Diskursbegriffes sichtbar, bei der sie das zitierte Bauarbeiterbeispiel inklusive dem Zusatz vom Sprachspiel als dem „Ganzen“ aufgreifen. Das, „was Wittgenstein ein Sprachspiel nennt“, so erklären sie, ist „wiederum [...] ein Beispiel für das, was wir Diskurs nennen“ (Laclau und Mouffe, 2006:145). Sie binden ihre Diskurskonzeption klar an Praxis. Mit Wittgenstein sehen sie die Bedeutung von etwas untrennbar mit dessen praktischer Verwendung verbunden. „That is to say in our terminology, every identity or discursive object is constituted in the context of an action." (Laclau, 1990:102). Das beziehen sie ausdrücklich auch auf nicht-sprachliche Tätigkeiten. Glasze und Mattissek stellen in diesem Zusammenhang fest, dass Laclau und Mouffe ,konsequenter als Foucault über den Bereich der Sprache hinaus[gehen]" (Glasze und Mattissek, 2009b:158). ${ }^{6}$ So betont Laclau: ,The distinction between linguistic and non-linguistic elements does not overlap with the distinction between ,meaningful ' and ,not-meaningful “.” (Laclau, 1990:102). Das Fragen nach einem Baustein oder das Heben eines Bausteins sind beides Teil eines Diskurses, verstanden als eine "meaningful totalit[y]" (ebd.).

\subsection{2 (Über)Determinierung, Bedeutungskonstitution durch Artikulation und Sedimentierungen}

Diese „meaningful totality“ ist nicht durch prädiskursive Strukturen determiniert, sondern entfaltet sich nur innerhalb von Praxis. Den Begriff der Überdeterminierung nutzen Laclau und Mouffe, um darauf hinzuweisen, dass Aspekte der sozialen Wirklichkeit nicht auf eine feststehende Bedeutung oder Ursache zurückzuführen sind, sondern das Bedeutung immer mannigfaltig, instabil und grundsätzlich offen ist (Laclau und Mouffe, 2006:186ff.) oder, mit Wittgenstein gesprochen, ,in der Luft“ hängt (s.o.). Beide DiskurstheoretikerInnen interessieren sich nun besonders dafür, wie sich Bedeutungsstrukturen herausbilden und sedimentieren. Ihre zentralen theoretischen Konzepte dafür sind Artikulation und leerer Signifikant. Jede Praxis ist insofern artikulatorisch, als sie einen Zusammenhang zu anderen (sprachlichen oder nicht-sprachlichen) Entitäten herstellt. Im Prozess der Artikulation, des In-Beziehung-Setzens, werden überdeterminierte Elemente zu (partiell) fixierten Momenten des Diskurses und erhalten so ihre Bedeutung. Durch „Abrichtung“ - Laclau und Mouffe selbst verwenden diesen Begriff nicht wiederholen sich die Artikulationen und bilden partiell verfestigte Strukturen.

\subsubsection{Ein-/Ausschluss und leere Signifikanten}

Artikulationen sind immer ein- und ausschließende Prozesse: Einschließend, weil sie Elemente In-Beziehung setzen und so integrieren - Laclau und Mouffe sprechen hierbei von Äquivalenzketten; ausschließend, weil die Elemente bestimmte/bestimmende Verknüpfungen eingehen und andere unwahrscheinlich machen. Dieses Prinzip machen sie auch für das Subjekt geltend. Subjektpositionen zeichnen sich durch bestimmte „Polsterknöpfe“ aus (z.B. Bauarbeiter, Mann, Stärke, Naivität, Authentizität) sind aber brüchig, insofern sich die Relationen verschieben können bzw. ein Subjekt sich, je nach Situation unterschiedlich, im Kreuzungspunkt verschiedener Identitäten temporär konstituiert (z.B.

\footnotetext{
${ }^{6}$ Glasze und Mattissek verweisen hier darauf, dass Foucaults Diskurskonzeptionen in dieser Dimension sehr uneindeutig sind, da er z.T. explizit diskursive von nicht-diskursiven Praktiken unterscheidet, an anderer Stelle diese Unterscheidung aber in Frage stellt.
} 
Bauarbeiter, Weißer, Deutscher, Vegetarier vgl. Glasze und Mattissek, 2009b:162f). ${ }^{7}$

Zentral für Laclau und Mouffe ist der Begriff leerer $\mathrm{Si}$ gnifikant. Dieser ist als ein (affektiv aufgeladener) symbolischer Bezugspunkt zu sehen (z.B. „Demokratie“), welcher die Konfigurierung von Äquivalenz- und Differenzbeziehungen und damit die symbolische Ordnung strukturiert. Als eine Art „kleinster gemeinsamer Nenner“ ist er in der Lage, viele verschiedene Momente (z.B. die Konzepte „Pressefreiheit“", ,Religionsfreiheit“”, ,Individualität“, ,,Marktwirtschaft" usw.) in eine Äquivalenzkette zu bringen und sie als Teilmomente (s)einer Identität begreifbar zu machen. Damit wird ein Zeichen wie z.B. „Demokratie“ leer, da es in der Bedeutung überdeterminiert ist. Es sind zahlreiche praktische Einsätze dieses Zeichens denkbar. In einem bestimmten praktischen Einsatz, wenn also in Bezug zu einem leeren Signifikanten situativ Sinn produziert wird, ist dieser dann aber temporär ,gefüllt". Der leere Signifikant ist als ein zentraler, wiederkehrender, unterschiedlich artikulierbarer Bezugspunkt in der Deutung von Welt, und damit als ein Knotenpunkt im „Weltbild“ (Wittgenstein), zu verstehen.

\subsubsection{Empirische Fokussierungen}

Laclau und Mouffe zeigen, wie Praktiken partiell und temporär „erstarren“ und als machtvolle „Leitungen“ für die soziale Orientierung fungieren. Diskursanalytisch wird der Fokus auf die Relationierung von (sprachlichen) Signifikanten gelegt. Das zeigt sich auch bei der methodischen Ausrichtung entsprechender humangeographischer Studien, die durch Verfahren wie Lexikometrie, Aussagenanalyse oder Kodieren Regelmäßigkeiten und Brüche der Artikulationen herausarbeiten. Der Analyseschwerpunkt liegt dabei häufig auf den ,größeren Zusammenhängen“. Laclau und Mouffe interessieren sich jenseits ihrer theoretischen Überlegungen weniger für die situative Verwobenheit von Praktiken auf Baustellen, sondern für eine Analyse der ,großen Erzählung" des Marxismus oder der Demokratie. Ähnliches gilt für viele der von Laclau und Mouffe inspirierten humangeographischen Arbeiten. In diachroner Perspektive werden die Entstehung und Wandlung von Diskursen über große Zeitspannen hinweg analysiert. Die niemals endgültig vollzogene Schließung von Bedeutung zeigt sich auf der empirischanalytischen Ebene in der Herausarbeitung von graduellen Verschiebungen zwischen verschiedenen historischen Phasen, die von jeweils unterschiedlichen hegemonialen Artikulationen geprägt sind (z.B. Wandel des Kultur-Diskurs der UNESCO, Dzudzek, 2013; Wandel des FrankophonieDiskurses, Glasze, 2013). Auch in synchroner Perspektive werden häufiger größere Maßstabsebenen angelegt, in-

\footnotetext{
${ }^{7}$ Mit Wittgenstein ausgedrückt: Das Subjekt folgt Regeln, nimmt so Subjektpositionen ein bzw. wird diesen zugewiesen (vgl. Althusser, 1977) und ist damit fähig, in verschiedenen Sprachspielen als eine bestimmte identitäre Handlungsinstanz aufzutreten.
}

dem Diskurse in unterschiedlichen gesellschaftlichen Kontexten betrachtet werden (z.B. die vergleichende Analyse von Großwohnsiedlungsdiskursen in Frankreich, Polen und (Ost-/West-)Deutschland, Brailich et al., 2008). Solche Arbeiten verdeutlichen eindrücklich die „Makrokonfiguration“ von Sprachspielen, für deren Analyse Laclau und Mouffe ein reiches theoretisches Vokabular zur Verfügung stellen.

\subsection{Die Praxistheorie Schatzkis}

Eine ähnliche Einschätzung dieser Diskurstheorie teilt auch Schatzki, wenn er den Ansatz als „Arrangement-Theorie“ bezeichnet (2002:50). Eingedenk dessen, dass Schatzki sein eigenes Theorieangebot um den Begriff practice-arrangementbundel aufbaut, deutet sich die Affinität sowie die unterschiedliche Schwerpunktsetzung beider Konzeptionen bereits an.

\subsubsection{Praktiken}

Seine praxistheoretischen Überlegungen bezeichnet Schatzki als einen „Wittgensteinan Approach“ (1996). Eine Praxis ist für den Sozialphilosophen ,a , bundle of activities', that is to say, an organized nexus of bodily doings and sayings" (Schatzki, 2002:71). Er nutzt den Begriff „bodily“, um zu betonen, dass Praktiken zunächst ,nichts anderes als Körperbewegungen darstellen“ und stellt diese ,scheinbare Trivialität" (Reckwitz, 2003:290) deshalb heraus, weil infolge des linguistic turns die Rolle des discourse im sozialen Leben überbewertet worden sei. Schatzki verwendet hier den Begriff discourse allerdings im Sinne von Sprache oder Text: sayings sind für ihn discursive, doings sind nondiscursive actions (Schatzki, 2002:76f.). Mit Wittgenstein geht es auch ihm darum „,das Ganze“ zu betrachten. „Practices are a motley of actions of both sorts, and it seems to me an error to grant priority to either type“" (ebd.:77).

\subsubsection{Praktische Intelligibilität und Organisationskomponenten von Praktiken}

Daneben ist für sein Praxisverständnis das Konzept der „,praktischen Intelligibilität“, verstanden als kontextabhängiges Weiterwissen, zentral (ebd.:74).

Um diese praktische Bedeutungskonstitution beschreibbar zu machen, unterscheidet er heuristisch vier zusammenhängende Organisationskomponenten von Praktiken. Das praktische Verstehen umfasst das know-how des Tuns (z.B. Wissen, wie man mauert), das know-how des Identifizierens (z.B. das Anmischen von Beton, Steineheben etc. als Hausbaupraxis zu begreifen) und das know-how des Reagierens/Antwortens (z.B. auf den Zuruf „Stein“ ein entsprechendes Objekt zu reichen). Verschiedene Aktivitäten werden auf ends hin ausgerichtet (z.B. Mauern, um einen Schutz zu haben). Er nennt diesen Aspekt Teleoaffektivität, um zu betonen, dass auch Gefühle eine wichtige Rolle spielen. Unter Regeln versteht 
er explizite, zu interpretierende Beschränkungen und Handlungsanleitungen (z.B. gesetzliche Vorgaben zur erlaubten Höhe einer Mauer). Und schließlich nennt Schatzki das allgemeine Verstehen als ,senses of the worth, value, nature, or place of things, which infuse and are expressed in people's doings and sayings" (Schatzki, 2012:16; z.B. dass man beim Hausbau ökologisch agiert).

\subsubsection{Sprachspiele als practice-arrangement-bunde/s}

Seit der Jahrtausendwende erweitert er seinen praxistheoretischen Blick mit dem Begriff der orders bzw. arrangements, verstanden als

arrangements of people, artifacts, organisms, and things through and amid which social life transpires, in which these entities relate, occupy positions, and possess meanings $[\ldots]$ in which nonhumans enjoy meaning, people assume meanings and identities, and entities of both sorts relate and occupy positions. (Schatzki, 2002:38)

Arrangements sind keine unabhängigen Konfigurationen, sondern existieren nur im Zusammenhang von Praktiken (ebd.:59). Folglich spricht Schatzki vom Sozialen als practice-arrangement-bundels, als Zusammenhang (des $\mathrm{Zu}-$ sammenhangs) von Praktiken und Arrangements.

Schatzkis Arrangementbegriff ist auch, das macht obiges Zitat deutlich, von Laclaus und Mouffes Diskurstheorie inspiriert. In beiden Denkgebäuden geht es um Konfigurationen, in denen verschiedene Entitäten praktisch zusammenhängen und sich in ihrer Verbindung bestimmen. Die Relationen, Positionen (z.B. Subjektpositionen) und Bedeutungen werden dabei als ,labile phenomena, only transitory fixations" (ebd.:31) gedacht.

Schatzki begründet die Integration des ArrangementBegriffs damit, dass er vorher die Rolle von Materialität nicht ausreichend beachtet hätte (ebd.:ix). Diese wird bei Laclau und Mouffe auch mitgedacht, nimmt aber analytisch einen untergeordneten Stellenwert ein. Sie fokussieren (symbolische) Relationen, Schatzki dagegen geht es stärker um das, was er „difference in use“ (ebd.:56) nennt, die (materiellen) Relationierungen, die in actu vollzogen werden.

\subsubsection{Empirische Fokussierungen}

Um seine theoretischen Überlegungen zu illustrieren, greift Schatzki u.a. auf das Beispiel einer religiösen ShakerCommunity zurück, die sich auf Heilmittelproduktion spezialisiert hat. Seine Ausführungen lesen sich als dichte Beschreibungen der materiellen Arrangements und der damit verwobenen Praktiken - z.B. wie sich verschiedene Praktiken um eine hydraulische Kräuterpresse organisieren (Schatzki, 2002). Für einen solchen Ansatz, ,the investigator has no choice but to do ethnography“" (Schatzki, 2012:24). Folglich legen auch die von Schatzki inspirierten Studien der Humangeographie einen entsprechenden methodischen Schwerpunkt. So situiert etwa Everts seine Studie über „Soziale Praktiken im multikulturellen Alltag“ (2009) in migrantengeführten Lebensmittelgeschäften und betrachtet diese als ein Produkt des „,fortwährende[n] Arrangieren[s] von Entitäten, bei dem sich Menschen, Artefakte, Dinge und Organismen begegnen und interagieren" (ebd.:285). Mithilfe teilnehmender Beobachtung und offener Interviewführung geht es darum, ein „,situierte[s] “ Vokabular“ und so die „Bedeutung der Geschäfte“ (ebd.:286) zu rekonstruieren. Dieses Vorgehen deuten wir als „Mikroarchitektur der Sprachspiele“. Anders ausgedrückt: Schatzkianische Analysen interessieren sich sehr wohl für Baustellen - und so ist es kein Zufall, dass der Sozialphilosoph ein Buch ,Site Ontology“ betitelte.

\section{4 Ähnlichkeiten, unterschiedliche Erkenntnisinteressen und Integrationspotential}

Für die beiden skizzierten Ansätze ist Wittgensteins „Gebrauchstheorie der Bedeutung“ zentral, die Bedeutung untrennbar an praktische Zusammenhänge bindet, „,das Ganze“ sprachlicher und nicht-sprachlicher Tätigkeiten bedenkt und die (In)Stabilität von Praxis betont. Jenseits der Praxis gibt es keine autonome, externe Struktur, die das Soziale determiniert. Beide Theorien folgen einer, bei Wittgenstein angelegten, „flachen Ontologie“ (Krämer, 2009:40), der gemäß soziale Realität nur auf einen Seinsbereich zurückzuführen ist.

Allerdings drücken sich in den diskurs- und praxistheoretischen Entwürfen auch unterschiedliche Erkenntnisinteressen aus. Laclau und Mouffe zeigen primär, wie sich verschiedene Elemente zu einer „meaningful totality“ verbinden und wie sich Bedeutungen, ausgedrückt in leeren Signifikanten, hegemonialisieren. Sie betrachten die Sedimentierungen und die ,unterschiedliche Zählebigkeit von materialzeichenhaften Entitäten“ (Van Dyk et al., 2014:352). Dabei betonen sie zwar die „Verflechtung von Sprache, Dingen, Handlungen und Welt" (ebd.), legen aber einen analytischen Schwerpunkt auf Fixierungen und Brüche, die sich in Weltbildern (z.B. Demokratie) zeigen und fokussieren damit die strukturierenden Funktion zentraler Symbole in Sprachspielen. Schatzki folgt der Aufforderung Wittgensteins - „Beschreibe Sprachspiele“ (Wittgenstein, 2008:§486; vgl. auch Wittgenstein, 1967:§299-314) - mit seinem ,primer on practices" (Schatzki, 2012) direkter. Sein Augenmerk liegt auf der minutiösen Beschreibung von Sprachspielen, stärker als materielle und situative practice-arrangement-bundels gedacht (z.B. Shaker-Community).

\section{Ergänzungs-/Integrationspotential}

Die diskutierten Familienähnlichkeiten liefern eine konzeptionelle Grundlage für ein Zusammendenken diskurs- und praxistheoretischer Herangehensweisen bei humangeographischer Forschung. Dabei kann die jeweils andere Theorie 
als ein familienähnliches Sensibilisierungsangebot gedacht werden. Wir möchten nun eine mögliche forschungsleitende Fragestellung für solche Unternehmungen diskutieren, die sich - in Anlehnung an einen Essaytitel von Laclau - wie folgt formulieren lässt: „How do empty signifiers matter?“8. Diese Frage betont die praktische Dimension der durch leere Signifikanten strukturierten symbolischen Ordnungen. Es geht darum, zu fragen, wie sie praktisch Bedeutung erlangen, insofern sie sich materialisieren (dass ,matter“ im Sinne von „Materie“ übersetzt werden kann ist durchaus gewollt). Durch welche Praktiken und aus welchen praktischen Kontexten heraus drückt sich eine bestimmte Materialität leerer Signifikanten aus? Inwiefern sind symbolische Bezugnahmen auf leere Signifikanten Momente eines stets komplexitätsreduzierenden „Weiterwissens“, das die doings und sayings organisiert und auf bestimmte ends hin ausrichtet? Inwiefern fungieren sie als situierte „Leitungen“ (Wittgenstein) und sind machtvoll, indem sie bestimmte Tätigkeiten integrieren und andere nicht. Die Wirkung leerer Signifikanten als gemeinschaftlich geteilte Bezugspunkte entsteht dabei gerade im Zusammenspiel aus Sprache, Körper und materiellem Kontext.

Bezüglich der verschiedenen Erkenntnisinteressen lässt sich die Frage nun spezifisch verorten. Grundsätzlich zeigt eine solche Perspektive auf, wie sich Praktiken mit differierendem Kontext beständig (graduell) verändern, gleichzeitig aber, um anschlussfähig zu sein, wiederkehrend auf ähnliche Art und Weise identifiziert werden müssen (Schäfer, 2013:311-327; mit Bezug zu Wittgensteins Philosophie auch Kripke, 1982:7-55). In diesem Zusammenhang ist mit Wittgenstein anzudenken, dass diese Identifizierungen als „ÜberAusdrücke“ (Wittgenstein, 2008:§189-192) wirken, indem sie sich als scheinbar feststehende, situationsübergreifende Identitäten/Kategorien präsentieren und somit verdinglichen. Wittgensteins Überausdrücke erinnern hierbei an leere Signifikanten sowie daran, dass jede Kategorie potentiell als leerer Signifikant wirken kann. Wittgenstein problematisiert, inwieweit (eigentlich) verschiedene Aktivitäten im Sprachspiel mit den gleichen Zeichen verstehbar gemacht werden (vgl. auch Schatzki (2002:12) über Wittgenstein). Während nun Schatzki jene ,verschiedenen Aktivitäten“ erfassen will und folglich Beobachtungs- und Analysekategorien entwickelt, um den Verdinglichungsprozessen von Zeichen/Kategorien nicht „aufzusitzen“, interessieren sich Laclau und Mouffe analytisch gerade für die komplexitätsreduzierende Dimension symbolischer Ordnungen, die jene vielschichtigen ,,verschiedenen Aktivitäten" sinnverknappend einordnen. Beide Interessen ergänzen sich dabei. Denn um die sinnverknappende Dimension von Praxis (hier: via der Verwendung leerer Signifikanten) herauszuarbeiten und in ihrer machtvollen Wirkung besser zu verstehen, ist die Rekonstruktion der praktischen Zusammenhänge/Sprachspiele nötig (Sprache,

\footnotetext{
${ }^{8}$ Im Original: „Why do empty signifiers matter to politics“ (Laclau, 1996:36-47).
}

Körper, Umgang mit Dingen, Architekturen), innerhalb derer dann Sinnverknappung als ein praktischer Prozess erst zu verorten ist. Es ist nötig, zu rekonstruieren, was die vielfältigen Aktivitäten ausmacht, die kategorisiert und ,zugeschnitten“ werden, um die machtvolle Funktion dieses „Ab- und Zuschneidens" besser zu verstehen.

\section{Empirische Beispiele}

Wir weiten unsere Anschlussstellenerkundung nun auf das empirische Terrain aus. Die folgenden Beispiele sind weniger als Darstellung empirischer Ergebnisse gedacht, sondern wollen die mögliche Befruchtung beider Theoriefamilien gegenstandsbezogen aufzeigen. Wenngleich die Beispiele unterschiedliche Momente einer integrativen Perspektive aufzeigen, eint sie der Fluchtpunkt auf die Frage „How do empty signifiers matter?“.

\subsection{Neue Ländlichkeit und Idyllisierung}

Auch im Zeitalter einer umfassenden Urbanisierung lässt sich eine Konjunktur des Ländlichen beobachten, die sich am eindrücklichsten in dem immensen Erfolg von Zeitschriften wie Landlust, Landidee oder mein schönes Land äußert. Trotz sinkender Verkaufszahlen von Printmagazinen, verkaufen sich diese Landzeitschriften immer mehr - allen voran die Landlust, die mittlerweile eine Käuferschaft von über einer Million/Ausgabe erreicht hat. Was bedeutet diese „Neue Landlust“? Mit Laclau und Mouffe lässt sich das „Ländliche“" als ein leerer Signifikant betrachten, der zahlreiche Äquivalenzbeziehungen herstellen kann. ${ }^{9}$ Durch eine Analyse der symbolischen Artikulationen, die in den Landmagazinen vollzogen sind (z.B. mittels kodierender Verfahren), ist es möglich, die „Ländlichkeit der Landmagazine“ systematisch zu rekonstruieren.

Die Magazine schließen an idyllische Repräsentationen (gedacht als Ergebnis medial-ökonomischer Repräsentationspraktiken) des Ländlichen an. Idyllen sind, gemäß Laclau und Mouffe'scher Ausrichtung, symbolische Konfigurationen von Ein- und Ausschlüssen. Das Ländliche wird als eine naturnahe, ursprüngliche, echte, entschleunigte/entschleunigende Lebensform an bestimmten Orten (für bestimmte Personen) artikuliert. U.a. folgende Aspekte sind an diesen Idyllen-Repräsentationen bemerkenswert. Erstens konzentrieren sie sich auf den ,eigenen Bereich“. Die referenzierten Orte sind weniger Orte des siedlungsstrukturellen ländlichen Raumes, sondern „Mikroorte“ der Alltagspraxis wie der eigene Garten, die eigenen vier Wände, etc. Zweitens, damit zusammenhängend, liegt ein Schwerpunkt der Magazine auf konkreten Praxistipps zu Bereichen wie Gärt-

\footnotetext{
${ }^{9}$ Dass Ländlichkeit ein widersprüchlicher leerer Signifikant ist, zeigt sich allein schon daran, dass es neben der Neuen Landlust natürlich auch sehr negative Semantisierungen des Ländlichen als rückständig, statisch etc. gibt (Redepenning, 2013).
} 
nern, Kochen, Handwerken, Dekoration, Naturerleben, etc. Dieses artikulierte Praktikenensemble bildet einen Hauptbestandteil der konstituierten Ländlichkeit. Die Praktiken lassen sich mit Reckwitz (2008) als ,ästhetisch“, als Praktiken der Gestaltung, sinnlichen Wahrnehmung und des Erlebens, charakterisieren. In dieser Weise führen die Zeitschriften eine „Ästhetisierung des Alltags“ (Schulze, 1992), eine Idyllisierung vor. Diese erscheint auf den ersten Blick als konflitklos oder apolitisch. Aber gerade weil die Idyllisierungen das Konflikthafte auszuschließen versuchen, weisen sie einen politischen Gehalt auf, insofern sie so eine Grenze zwischen der Alltagsästhetik des eigenen Bereichs zu einem antagonistischen Außen ziehen und damit das Soziale zu ordnen versuchen.

Die Zeitschriften sind in zweifacher Weise praktisch. Sie sind Repräsentationen einer ,praktischen Ländlichkeit“. Ebenso sind sie ein Artefakt und somit Teil eines practicearrangement-bundels, zu dem mediale Produktionspraktiken wie auch die Konsumption und damit verbundene Praktiken gehören. Um zu zeigen, ,how this new rurality matters?“, ist gerade der Blick auf die Dimension der Alltagspraxis, auf die situativen Idyllisierungen, vielversprechend.

Auf methodischer Ebene betrifft eine solche Ergänzung die Einbeziehung ethnographisch informierter Verfahren. Dies ist bei dem Forschungsobjekt Landmagazine nicht unproblematisch, sind das Lesen der Magazine wie auch die „Alltagspraktiken des eigenen Bereichs“ Vorgänge, zu denen man kaum unmittelbaren Forschungszugang bekommt. Eine Umgangsweise mit diesem Problem sind im ,eigenen Bereich" situierte, kontextsensible und ausgiebige Interviews, bei denen auch die Magazine vorgelegt werden und die Möglichkeit besteht, sich als wichtige darstellende Aspekte vergleichsweise unmittelbar zeigen zu lassen. So lassen sich verschiedene Facetten der alltäglichen Idyllisierung rekonstruieren.

Ein wichtiger Aspekt dieser Idyllisierung ist die (körperliche) Praxis des Lesens. Die LeserInnen begreifen ihre Lektuire als Bruch, als Moment des Aussteigens aus dem sonst üblichen Lebensrhythmus. Man nimmt sich Zeit, man versucht das Lesen in ein solches Arrangement einzupassen, bei dem man möglichst ungestört ist. Eine Interviewpartnerin schildert beispielsweise, wie sie sich ihre Lektüre versucht aufzusparen, bis sie Zeit für ein warmes Bad findet. Rezeptionsorte wie Badezimmer, aber auch Gartenterrasse oder Schlafzimmer, zeigen eine exkludierende Dimension der Idyllisierung auf. Die Praktiken sind selbstbezogen, im Privaten situiert und auf das Private ausgerichtet. Das gilt nicht nur für das Lesen, sondern auch für die Bewertung der in den Magazinen referenzierten Praktiken, die für die Leser als etwas „wo man sich wegträumen kann“ oder als offene Vorlagen, mit denen man ,auch was macht“, aufgefasst werden. Dieses Machen betrifft zahlreiche, je nach konkreten Lebensumständen variierende Aspekte, vom Verschönern des eigenen Bereichs zur aktiven Auseinandersetzung mit der Natur (bevorzugt im heimischen Garten) und ist somit anschlussfähig an zahlreiche Alltagspraktiken. Die Zeitschriften bedeuten für die LeserInnen Anregungen, ihr Leben „ländlicher“ zu gestalten. Der Wohnort ist dabei sekundär. Das Leben im eigenen Zuhause wird ruralisiert, es wird zu ,mein[em] Stück Land“, wie es ein Interviewpartner, der im Frankfurter Süden lebt, ausdrückt. Verschiedene Alltagspraktiken wie Gärtnern, Einkaufen, Handwerken etc. organisieren sich partiell und temporär zu einem Idyllisierungsensemble und erfahren eine bestimmte (gerade auch teleoaffektive) Akzentuierung. Beispielsweise das Gärtnern, als eine Facette der Idyllisierung/Verländlichung artikuliert, wird weniger als Moment der „nötigen Arbeit“ oder der „,reinen Zierde“ praktiziert, sondern als Moment einer aktiven, ästhetischen und ungestörten Naturerfahrung, deren Erleben im Gegensatz zum „sonstigen Leben“ steht.

Wie in diesem Beispiel angedeutet, kann die diskutierte Forschungsausrichtung konzeptionell begründet herausarbeiten, wie sich der leere Signifikant Ländlichkeit in bestimmten Kontexten praktisch füllt. Eine solche Analyse der „Neuen Landlust“ ist eine detaillierte „Füllungsstudie“, die sich durch die Betrachtung weiterer „Sprachspiele“, in denen und durch die Ländlichkeit relevant wird (z.B. bei Raumplanung, lokalpolitischen Konflikten), weiter kontextualisieren ließe.

\subsection{Rap und stigmatisierte Banlieues}

Eine weitere Illustration der diskutierten Aspekte erfolgt am Beispiel von französischem Rap zu stigmatisierten Vierteln. Rap bietet als „Musik der Viertel“ gegenhegemoniale Perspektiven auf die Verhandlung von Stigmatisierung (Germes und Tijé Dra, 2012). Die Sprachspiele im Rap konstituieren - Regelhaftigkeiten der Sagbarkeit und Darbietung folgend mehrere heuristisch definierbare Bereiche. Eine Relationierung dieser Bereiche betont ihre stabilen als auch instabilen Verwobenheiten und spürt der Organisation gegenhegemonialer Momente in (situierten) Sprachspielen nach.

Den naheliegendsten Forschungsbereich bilden Raptexte. Diskursanalytische Verfahren zeigen, wie über sozialräumliche Dichotomien gesellschaftskritische banlieueRepräsentationen artikuliert werden (genauer: Tijé-Dra, 2014a, b). Dabei zeigt sich u.a., dass der leere Signifikant „Autonomie“ eine wichtige Rolle spielt. Autonomie markiert hier Abgrenzung und Unabhängigkeit von einem antagonistischen Außen, bestehend aus ,den staatlichen Institutionen“, „den Medien“ oder „den dominierenden Gesellschaftsschichten“.

Daneben geben auch die Darbietungsformen von Rap Forschungsbereiche vor. Bei Konzerten oder RapSchreibwerkstätten (Ateliers) kann Sprachspielen in situ nachgespürt werden. In diesen Bereichen reproduzieren sich gegenhegemoniale Diskurse in unterschiedlichsten materiellen Arrangements. Ateliers sind sozialpädagogische Projekte, die in Gemeinschaftsräumen von kommunalen Jugendzentren stigmatisierter Viertel situiert sind. Jugendliche 
üben sich unter Anleitung im Raptexte schreiben, benutzen Reimwörterbücher und tragen ihre Ergebnisse vor. Angeleitet von RapperInnen, bekommen sie die Konventionalitäten des Sprachgebrauchs sowie seiner Darbietung teilweise neu gelehrt.

Solche Projekte transferieren bestimmte Sprachspiele in ein von üblichen Darbietungsformen abweichendes Arrangement: kommunale Jugendzentren unterscheiden sich deutlich von Szeneclubs oder sonstigen Konzertstätten (z.B. Zielgruppe, Architektur, Körperpositionierung).

Durch Konsum und Imitation von Rap-Musik verfügen die Jugendlichen bereits über ein ausgerichtetes, inkorporiertes knowing-to, das in die Ateliers mit ,hineingetragen“ wird: Begriffswahl, Kleidungsstil, Haltung, Gesten, Vorstellungen von ,gutem Rap“. Auch die (Rap-)LeiterInnen der Ateliers bringen praktisch erlernte Ausdrucksweisen mit an den ,neuen“ Ort. In diesem Zusammenhang ist gerade die Deutungspraxis bezüglich jener kommunal geförderten Ateliers instabil (gegenüber der „üblichen“ Aufführungsorte von Rap). Je nach Standpunkt bewerten Szene-Akteure die Ateliers zwischen begrüßenswerter Jugendarbeit und institutioneller Vereinnahmung (Lafargue, 2008). Die RapperInnen müssen symbolische Ordnungen gegenhegemonialer Diskurse mit weiteren Vollzugskontexten in Einklang bringen, wenn ihre Praxis „autonom“ oder „kritisch“ ausgerichtet sein soll. „Unähnliche“ "Sprachspiele bedürfen ,sinnvoller“ Integration in die bisherige künstlerische Praxis. Der Bezug auf leere Signifikanten kann dabei eine „kittende“ Wirkung erzielen. Ein kurzer Interviewausschnitt mit Skalpel, Rapper aus SaintDenis, soll das verdeutlichen. Skalpel praktiziert einen anarchistischen, militanten Rap, bei dem „Autonomie“ besonders betont wird. Wie macht er sein Engagement innerhalb eines kommunalen Kontext sinnhaft, wie handhabt er die Reibungspunkte zwischen den Subjektpositionen eines ,kritischen Rappers“ und eines „Anleiters“ in kommunalen und pädagogischen Arrangements? Rap-Ateliers in der Stadtteilarbeit findet er aufgrund der Popularität von Rap schlüssig. Die beteiligten Institutionen versuchten jedoch,

Rap zu besänftigen - jedenfalls zu vereinnahmen - der eine legitime Revolte ausdrücken könnte. Sie [...] ermöglichen [...] aber mit der Bereitstellung von Zubehör Rap zu machen, um dann zu sagen: „Seht her, wir sind gar nicht so böse weil wir Studio und Ausrüstung bereitstellen “ [...] Daher ist einer meiner Kämpfe, den Leuten zu sagen, dass sie so autonom wie möglich sein sollten. Es lohnt sich eher, 100 Euro zu sparen. Dafür kann man was kaufen und ist unabhängig, anstatt mit der Verwaltung eine Partnerschaft zu schließen.

Während Skalpel eine mangelnde Anerkennung politisierter Rap-Musik konstatiert, unterstellt er der Verwaltung, Rap als eine „,besänftigende“ Beschäftigungsmöglichkeit auszunutzen. Neben einer Schwächung des symbolischen Gehalts von Rap-Praktiken gehen die Ateliers - als spezifisches
Sprachspiel denkbar - gemäß Skalpel mit neuen Abhängigkeiten und Machteffekten einher. Um diesen Effekten zu entfliehen rät er, Studiotechnik selbst zu erwerben. Er zitiert hier den im gegenhegemonialen Diskurs zentralen, leeren Signifikanten der „Autonomie“. Auf bestimmte Weise schafft es Skalpel dennoch, eine Tätigkeit in pädagogischen Ateliers mit seiner Deutung eines gegenhegemonialen Regelfolgens zu vereinen. Er könne ,seine“ Botschaft platzieren. Maßgebend dabei ist vom „offiziellen Skript“ abweichen zu können, um in situ Bedeutungsverschiebungen zu bewirken:

Stell dir vor du wärst ein Geschichtslehrer, der Kinder nach Lehrplan unterrichtet [...] Du wirst nach deiner subjektiven Art als aktivistischer Lehrer - oder auch nicht - ein Thema so präsentieren, dass das Kind nicht nur eine offizielle Version hört, sondern verschiedene.

Skalpel versucht Irritationen an seiner Tätigkeit zuvorzukommen. Er mobilisiert das Konzept des „Lehrers“ und sieht sich damit bestimmten Regeln folgen (allen voran: ,autonomes Denken befördern“). Diese erkennt er als anschlussfähig sowohl an die Regelhaftigkeiten des Rapper-seins (,Autonomie") als auch an die Regelhaftigkeiten des Sprachspiels kommunal geförderter Rap-Ateliers (,Lehrer-sein“). „Autonomie“ wird hier zu etwas, das ein „Lehrer“ den „Schülern“ im Rap-Atelier beibringen kann. Der Signifikant „Autonomie" materialisiert sich im Zusammenspiel aus Deutungspraktiken, Subjektivitäten und materiellen Arrangements und schafft es, verschiedene Praktiken zu Momenten seiner Identität zu machen: Skalpel bleibt ,kritischer Rapper“ als auch „Kommunalarbeiter“ im Atelier. Diese Position gibt ihm die Möglichkeit, bestimmte gegenhegemoniale Momente, wie etwa die der „legitimen Kritik“, in die Ateliers zu integrieren und an Jugendliche heranzutragen. Andernfalls würde auch er keine Ateliers durchführen:

Ich leite die Schreibwerkstatt nach meiner subjektiven Art, d.h. ich werde [...] den Jugendlichen die Möglichkeit einräumen sich frei auszudrücken, ohne sie dabei belehren zu wollen [...]. Das ist eine unverhandelbare Bedingung für mich.

Anhand der Passagen, in denen ein „Praktiker“ konfliktuelle artikulatorische Praxis explizit macht, wird deutlich, wie widersprüchlich, umkämpft, aber auch kreativ die Reproduktion gegenhegemonialer Diskurse und ihrer Sprachspiele verläuft. Das Beispiel zeigt, wie die Frage, ob man einer Regel folgt oder nicht (mehr), immer kontextuell ausgehandelt wird. Für die in diesem Aufsatz skizzierte Perspektive sind die Momente der Brüche und Konflikte interessant, in denen eine explizite Verhandlung von Regelfolgen aufscheint. Die Fragen sind dann: Wie und in welchen Kontexten werden solche Konflikte mit Bezug auf bestimmte Deutungen (leere Signifikanten) geglättet, d. h., so umgedeutet, dass sie keine Konflikte mehr darstellen? Und in welchen praktischen $\mathrm{Zu}-$ sammenhängen ist dies aber nicht mehr möglich? Um zu ver- 
stehen, wo im Verlauf sozialer Praxis Irritationen überhaupt aufscheinen und wie sie dann verhandelt werden (können), ist ein Verständnis des organisierten Ablaufs sozialer Praxis notwendig.

\section{Fazit}

Die Konzeptualisierung des Verhältnisses von Diskurs und Praxis ist komplex. Im Vokabular Laclaus und Mouffes ausgedrückt weist es Potential für dichotomische Relationierungen auf, bei denen jeweils entsprechende Äquivalenzketten und Antagonismen artikuliert werden (z.B. Diskurs: sprachlich, immateriell, abstrakt, textuell; Praxis: nicht-sprachlich, materiell, konkret, kontextuell). Diese können sich - mit Wittgenstein und Schatzki als difference in use gedachten im sozialwissenschaftlichen Arbeiten (von Forschungspraktiken bis hin zu strategischen Positionierungspraktiken) aktualisieren und, sofern sich dieser Antagonismus sedimentierte, $\mathrm{zu}$,,anderen Schulen“ führen.

Angeregt von aktuellen Debatten der Kultur- und Sozialgeographie hat unser Beitrag versucht, das vermeintlich „Andere“ mitzudenken. Mit dem Bezug auf die bislang eher unabhängig voneinander diskutierten Denkgebäude Laclaus und Mouffes sowie Schatzkis ging es uns darum, „,offene Türen" zu suchen. Wittgenstein, der für beide Theorien jeweils Teile des Bauplans beigesteuert hat, lieferte uns hierfür den Wegweiser und ließ uns fündig werden (zusammenfassend in Kap. 3.4).

Die erarbeiteten Anschlussstellen sensibilisieren praxistheoretische Arbeiten nach Schatzki für einen analytischen Blick auf „Weltbilder“, die situativ vollzogenen Praktiken inhärent sind und diese intelligibel machen. Das diskutierte praxeologische Vokabular liefert wiederum Analysekategorien, die einen differenzierten Blick auf die Mikroarchitektur und Organisation von Praktiken erlauben. Der diskurstheoretische Diskussionszusammenhang kann hiervon entsprechend profitieren, als mit Einbezug dieser Beobachtungsund Analysekategorien differenzierter rekonstruiert werden kann, wie symbolische Ordnungen in Praktiken ihren Niederschlag finden. Es lässt sich herausarbeiten, welche Praktiken und auch - mikroanalytisch(er) - welche Komponenten von Praktiken (vgl. Kap. 3.2) auf bestimmte Identitäten/Ziele hin ausgerichtet werden und im Kontext welcher Praktiken sich bestimmte symbolische Ordnungen auch brechen und partiell verändern können.

Die Frage „How do empty signifiers matter?“" erscheint uns als ein produktiver Fluchtpunkt für eine integrative Perspektive zwischen Diskurs und Praxis. Zwei erkundende Beispiele illustrierten Anwendungsmöglichkeiten dieser Perspektive für empirische Forschung. Das erste Beispiel zeigte, wie der leere Signifikant „Ländlichkeit“ im Rahmen der „Neuen Landlust“" praktisch gefüllt wird, das zweite verdeutlichte, wie „Autonomie“ als ein zentraler Bezugspunkt gegenhegemonialer Rap-Diskurse im „unüblichen“ Rahmen staatlich geförderter Schreibateliers in stigmatisierten Stadtteilen integriert wird.

Danksagung. Wir bedanken uns recht herzlich für die hilfreichen Kommentare und Anregungen, die wir im Verlauf der Ausarbeitung dieses Manuskripts erhalten haben. Unser Dank gilt im Besonderen Jonathan Everts und Tim Elrick sowie den drei anonymen GutachterInnen

Edited by: B. Korf

Reviewed by: three anonymous referees

\section{Literatur}

Althusser, L.: Ideologie und ideologische Staatsapparate, Aufsätze zur marxistischen Theorie, VSA, Hamburg/Berlin, 1977.

Angermüller, J., Nonhoff, M., Herschinger, E., Macgilchrist, F., Reisigl, M., Wedl, J., Wrana, D., und Ziem, A. (Hrsg.): Diskursforschung: Ein interdisziplinäres Handbuch, 2 Bände, Transcript, Bielefeld, 2014.

Bloor, D.: Wittgenstein and the priority of practice, in: The practice turn in contemporary theory, Herausgeber: Schatzki, T., KnorrCetina, K., und Von Savigny, E., Routledge, London/New York, 103-115, 2001.

Brailich, A., Germes, M., Schirmel, H., Glasze, G., und Pütz, R.: Die diskursive Konstitution von Großwohnsiedlungen in Frankreich, Deutschland und Polen, Europa Regional, 16, 113-128, 2008.

Bröckling, U., Krasmann, S., und Lemke, T. (Hrsg.): Gouvernementalität der Gegenwart, Studien zur Ökonomisierung des Sozialen, Suhrkamp, Frankfurt am Main, 2000.

Bührmann, A. D. und Schneider, W.: Vom Diskurs zum Dispositiv, Transcript, Bielefeld, 2008.

Diaz-Bone, R.: Kulturwelt, Diskurs und Lebensstil. Eine diskurstheoretische Erweiterung der Bourdieuschen Distinktionstheorie, Deutscher Universitätsverlag, Wiesbaden, 2010.

Dzudzek, I.: Hegemonie kultureller Vielfalt: Eine Genealogie kultur-räumlicher Repräsentationen der UNESCO, LIT Verlag, Berlin/Münster, 2013.

Everts, J.: Soziale Praktiken im multikulturellen Alltag. Bedeutungen migrantengeführter Lebensmittelgeschäfte, Berichte zur deutschen Landeskunde, 83, 281-296, 2009.

Everts, J., Lahr-Kurten, M., und Wattson, M.: Practice matters! Geographical inquiry and theories of practice, Erdkunde, 65, 323-334, 2011.

Foucault, M.: Archäologie des Wissens, Suhrkamp, Frankfurt am Main, 1973.

Foucault, M: Wie wird Macht ausgeübt?, in: Michel Foucault: Jenseits von Strukturalismus und Hermeneutik, Herausgeber: Dreyfuß, H. L. und Rabinow, P., Athenäum, Frankfurt am Main, 251261, 1987.

Germes, M. und Tijé-Dra, A.: Banlieue, in: Ortsregister. Ein Glossar zu Räumen der Gegenwart, Herausgeber: Marquardt, N. und Schreiber, V., Transcript, Bielefeld, 32-38, 2012.

Glasze, G.: Politische Räume. Die diskursive Konstitution eines "geokulturellen Raums" - die Frankophonie, Transcript, Bielefeld, 2013. 
Glasze, G. und Mattissek, A.: Diskursforschung in der Humangeographie: Konzeptionelle Grundlagen und empirische Operationalisierungen, in: Handbuch Diskurs und Raum: Theorien und Methoden für die Humangeographie sowie die sozial- und kulturwissenschaftliche Raumforschung, Herausgeber: Glasze, G. und Mattissek, A., Transcript, Bielefeld, 11-59, 2009a.

Glasze, G. und Mattissek, A.: Die Hegemonie- und Diskurstheorie von Laclau und Mouffe, in: Handbuch Diskurs und Raum: Theorien und Methoden für die Humangeographie sowie die sozial- und kulturwissenschaftliche Raumforschung, Herausgeber: Glasze, G. und Mattissek, A., Transcript, Bielefeld, 153179, 2009b.

Glasze, G. und Mattissek, A.: Diskursforschung in der Humangeographie, in: Diskursforschung: Ein interdisziplinäres Handbuch. Band 1: Theorien, Methodologien und Kontroversen, Herausgeber: Angermüller, J., Nonhoff, M., Herschinger, E., Macgilchrist, F., Reisigl, M., Wedl, J., Wrana, D., und Ziem, A., Transcript, Bielefeld, 208-223, 2014.

Glasze, G. und Pütz, R.: Sprachorientierte Forschungsansätze in der Humangeographie nach dem linguistic turn, Geogr. Z., 95, 1-4, 2007.

Jäger, S.: Text- und Diskursanalyse. Eine Anleitung zur Analyse politischer Texte, DISS, Duisburg, 1989.

Jäger, S.: Diskurs und Wissen - Theoretische und methodische Aspekte einer Kritischen Diskurs- und Dispositivanalyse, in: Handbuch sozialwissenschaftliche Diskursanalyse, 3. Aufl., Herausgeber: Keller, R., Hirseland, A., Schneider, W., und Viehöver, W., VS Verlag für Sozialwissenschaften, Wiesbaden, 91-124, 2011.

Keller, R.: Diskursforschung, VS Verlag für Sozialwissenschaften, Wiesbaden, 2011a.

Keller, R.: Wissenssoziologische Diskursanalyse. Grundlegung eines Forschungsprogramms, VS Verlag für Sozialwissenschaften, Wiesbaden, 2011b.

Krämer, S.: Von der „Tiefe“ des intellektualistischen Sprachbildes zur „Oberfläche“ der verkörperten Sprache, in: Oberfläche und Performanz. Untersuchungen zur Sprache als dynamische Gestalt, Herausgeber: Linke, A. und Feilke, H., Max Niemeyer, Tübingen, 33-50, 2009.

Kripke, S. A.: Wittgenstein on rules and private language. An elementary exposition, Harvard University Press, Harvard, 1982.

Laclau, E.: New Reflections on the Revolution of Our Time, Verso, London/New York, 1990.

Laclau, E.: Emancipation(s), Verso, London/New York, 1996.

Laclau, E. und Mouffe, C.: Hegemonie und radikale Demokratie. Zur Dekonstruktion des Marxismus, Passagen Verlag, Wien, 2006.

Lafargue, L.: Politique du hip-hop: Action publique et cultures urbaines, Socio-logiques, Presses universitaires du Mirail, Toulouse, 2008.

Link, J.: Kollektivsymbolik und Mediendiskurse. Zur Aktuellen Frage, wie subjektive Aufrüstung funktioniert, kultuRRevolution, 1, 6-21, 1982.

Lorimer, H.: Cultural geography: the busyness of being "more-thanrepresentational“, Prog. Hum. Geog., 29, 83-94, 2005.

Miggelbrink, J. und Schlottmann, A.: Diskurstheoretisch orientierte Analyse von Bildern, in: Handbuch Diskurs und Raum: Theorien und Methoden für die Humangeographie sowie die sozial- und kulturwissenschaftliche Raumforschung, Herausgeber: Glasze, G. und Mattissek, A., Transcript, Bielefeld, 181-198, 2009.

Mouffe, C.: Das demokratische Paradox, Turia + Kant, Wien, 2008.

Müller, M.: Reconsidering the concept of discourse for the field of critical geopolitics. Towards discourse as language and practice, Polit. Geogr., 27, 322-338, 2008.

Müller, M.: Making great power identities in Russia, LIT Verlag, Münster, 2009.

Puhl, K.: Regelfolgen, in: Philosophische Untersuchungen, Herausgeber: Savigne, E., Ludwig Wittgenstein, Akademie Verlag, Berlin, 91-109, 2011.

Reckwitz, A.: Grundelemente einer Theorie sozialer Praktiken: Eine sozialtheoretische Perspektive, Z. Soziol., 32, 282-301, 2003.

Reckwitz, A.: Elemente einer Soziologie des Ästhetischen, in: Unscharfe Grenzen. Perspektiven der Kultursoziologie, Herausgeber: Reckwitz, A., Transcript, Bielefeld, 259-282, 2008.

Redepenning, M.: Neue Ländlickeit, in: Europa - eine Geographie, Herausgeber: Gebhard, H., Glaser, R., und Lentz, S., Spektrum, Berlin/Heidelberg, 412-414, 2013.

Römpp, G.: Ludwig Wittgenstein, Köln, UTB, 2010.

Schäfer, H.: Die Instabilität der Praxis: Reproduktion und Transformation des Sozialen in der Praxistheorie, Velbrück Wissenschaft, Weilerswist, 2013.

Schatzki, T.: Social practices: A Wittgensteinian approach to human activity and the social, Cambridge University Press, New York, 1996.

Schatzki, T. R.: The site of the social: A philosophical account of the constitution of social life and change, Pennsylvania State University Press, University Park, 2002.

Schatzki, T.: A primer on practices, in: Practice-based education. Perspectives and strategies, Herausgeber: Higgs, J., Barnett, R. Billett, S., Hutchings, M., und Trede, F., Sense Publishers, Rotterdam/Boston, 13-26, 2012.

Schulte, J.: Wittgenstein. Eine Einführung, Reclam, Leipzig, 2001. Schulze, G.: Die Erlebnisgesellschaft. Kultursoziologie der Gegenwart, Campus Verlag, Frankfurt, 1992.

Strüver, A. und Wucherpfennig, C.: Performativität, in: Handbuch Diskurs und Raum: Theorien und Methoden für die Humangeographie sowie die sozial- und kulturwissenschaftliche Raumforschung, Herausgeber: Glasze, G. und Mattissek, A., Transcript, Bielefeld, 107-127, 2009.

Thrift, N.: Non-representional Theory, Routledge, Abingdon, 2008.

Tijé-Dra, A.: Rapper vs. Polizei? Zu einem ,französischen Verhältnis“, suburban, 2, 131-136, 2014a.

Tijé-Dra, A.: Eine andere banlieue? Eine Diskursanalyse gegenhegemonialer Raumproduktionen, Europa Regional, 20, 2-3, 2014b.

Van Dyk, S., Langer, A., Macgilchrist, F., Wrana, D., und Ziem, A.: Discourse and beyond? Zum Verhältnis von Sprache, Materialität und Praxis, in: Diskursforschung: Ein interdisziplinäres Handbuch. Band 1: Theorien, Methodologien und Kontroversen, Herausgeber: Angermüller, J., Nonhoff, M., Herschinger, E., Macgilchrist, F., Reisigl, M., Wedl, J., Wrana, D., und Ziem, A., Transcript, Bielefeld, 347-364, 2014.

Wittgenstein, L.: Zettel, in: Ludwig Wittgenstein: Zettel, Herausgeber: Anscombe, G. E. M. und Von Wright, G. H., University of California Press, Los Angeles, 1967.

Wittgenstein, L.: Über Gewissheit, Suhrkamp, Frankfurt am Main, 1970. 
Wittgenstein, L.: Philosophische Untersuchungen, 4. Aufl., Suhrkamp, Frankfurt am Main, 2008.

Wolkersdorfer, G.: Politische Geographie und Geopolitik zwischen Moderne und Postmoderne, Heidelberger Geographische Arbeiten 111, Heidelberg, 2001.

Wrana, D.: Diesseits von Diskursen und Praktiken. Methodologische Bemerkungen zu einem Verhältnis, in: Feld und Theorie. Herausforderungen erziehungswissenschaftlicher Ethnographie, Herausgeber: Friebertshäuser, B., Kelle, H., Boller, H., Bollig, S., Langer, A., und Ott, M., Barbara Budrich, Opladen, 185-200, 2012.

Wrana, D. und Langer, A.: An den Rändern der Diskurse. Jenseits der Unterscheidung diskursiver und nicht-diskursiver Praktiken, in: Forum Qualitative Sozialforschung (FQS), 8 (2), Art. 20, 2007.
Wrana, D. und Ott, M.: Gouvernementalität diskursiver Praktiken. Zur Methodologie der Analyse von Machtverhältnissen am Beispiel einer Massnahme zur Aktivierung von Erwerbslosen, in: Diskursanalyse meets Gouvernementalitätsforschung. Perspektiven auf das Verhältnis von Subjekt, Sprache, Macht und Wissen, Herausgeber: Angermüller, J. und van Dyk, S., Campus Verlag, Frankfurt, 155-182, 2010.

Wucherpfennig, C. und Strüver, A.: „Es ist ja nur ein Spiel...“ Zur Performativität geschlechtlich codierter Körper, Identitäten und Räume, Geogr. Z., 102, 175-189, 2014. 